\title{
FAINT GALAXY NUMBER COUNTS
}

\author{
T. Shanks, P.R.F. Stevenson and R. Fong \\ Physics Department, University of Durham, England \\ H.T. Mac Gillivray, \\ Royal Observatory, Edinburgh.
}

\begin{abstract}
Using photographic data obtained from the U.K. Schmidt and AngloAustralian telescopes we have determined galaxy number counts in the range $17^{\mathrm{m}} \leqslant B \leqslant 23^{\mathrm{m}}$ and $15^{\mathrm{m}} \leqslant \mathrm{R} \leqslant 22^{\mathrm{m}}$. By comparing our observations with models we find that the blue counts show strong evidence for galaxy luminosity evolution. We find that the red counts are better fitted by non-evolving models and that, here, the effects of the cosmological deceleration parameter, $\mathrm{q}_{0}$, and luminosity evolution are comparable. We discuss the implications of these results, with particular reference to the prospects of using the red counts to obtain constraints on $q_{0}$.
\end{abstract}

\section{INTRODUCTION}

The galaxy number-magnitude relation $n(m)$, contains much information on the luminosity evolution of galaxies at high redshift. This information is important because, unlike other probes, $n(m)$ is sensitive to evolution in the absolute luminosity of galaxies as well as to evolution in their colours. Over the years many attempts have been made to determine $\mathrm{n}(\mathrm{m})$ but its observed form is still not well established. Here we report on a new attempt to determine $n(m)$ in a blue $\left(b_{J}\right)$ and a red $\left(r_{F}\right)$ passband from photographic data at the South Galactic Pole (SGP). A more detailed account of this work is given by Shanks et al. 1984 (henceforth SSFM).

To interpret galaxy counts it is important to know the form of $n(m)$ at bright as well as faint magnitudes. We therefore began our study by obtaining COSMOS (Stobie et al. 1979) machine measurements of blue and red U.K. Schmidt photographs of a 12 square degree area at the SGP. The measurements defined $\mathrm{n}(\mathrm{m})$ in the range $17^{\mathrm{m}}-\mathrm{b}_{\mathrm{J}}<20 \mathrm{~m} .5$. We then also obtained COSMOS measurements of AAT photographs of 0.4 . square degrees of sky at the centre of the Schmidt photograph. These defined our $n(m)$ relation in the range $20 \mathrm{~m}_{5}<\mathrm{b}_{\mathrm{J}} \leqslant 23^{\mathrm{m}}$. 
In our photometry we employ isophotal magnitudes with isophotes set at $1-2 \%$ of sky for faint galaxies. We have verified our photometry and star-galaxy separation techniques on the UKST plates by comparing with the PDS data of the Oxford group (Fong et al. 1983). MacGillivray and Dodd (1982) have verified our photometry techniques at the faint limits of the AAT plates by comparison with the faint photographic photometry of Carter (1980). A new check on the faint AAT photometry has recently been made possible by the obtaining of CCD photometry on the SGP field by Couch et al. 1984. The comparison of the red photographic and CCD magnitudes is shown in Figure 1 where it can be

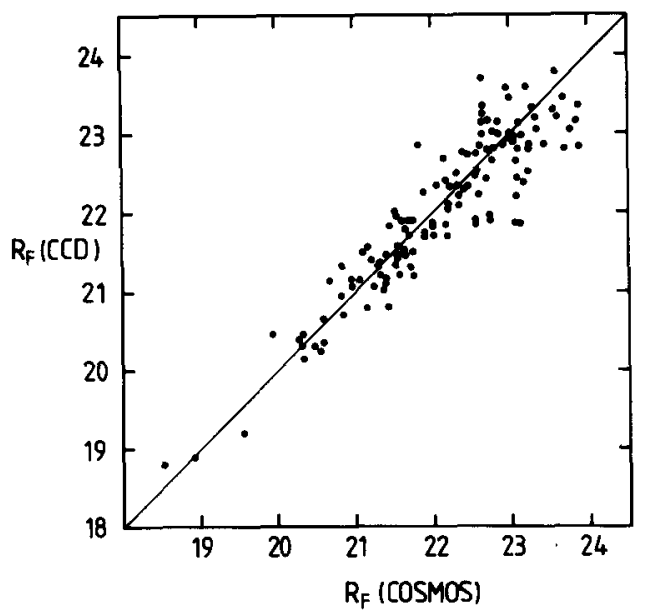

Figure 1 .

Comparison between COSMOS photographic galaxy photometry and the CCD photometry of Couch et a1., 1984.

seen that there is good agreement in zeropoint, with no evidence of scale errors in our photometry. Similarly good agreement is found for the blue magnitudes, when account is taken of the different photographic and $\mathrm{CCD}$ blue passbands. It is interesting to note that the CCD magnitudes were produced using the algorithms of Couch and Newell (1984), which are quite independent of those used to produce the photographic photometry. These algorithms produce pseudototal magnitudes like those used by Kron (1978) and the absence of any scale error in Figure 1 suggests that our isophotal magnitudes are very close to total magnitudes in the range of interest here.

\section{OBSERVED COUNTS}

Our observed counts in the blue and red passbands are shown in Figures $2 a, b$. The circles represent the UKST counts, the triangles the AAT counts. In the fainter magnitude range SSFM made a detailed comparison of these results with other authors. Briefly they found that in the blue there was excellent agreement at faint limits with the counts of Kron (1978), Koo (1981) and Couch and Newell (1984). Less good agreement was found with the counts of Tyson and Jarvis (1979) and Peterson et al. (1979) which seem to be shifted faintwards by $\sim 0 \mathrm{~m}_{5}$ with respect to our data. It is difficult to believe that 

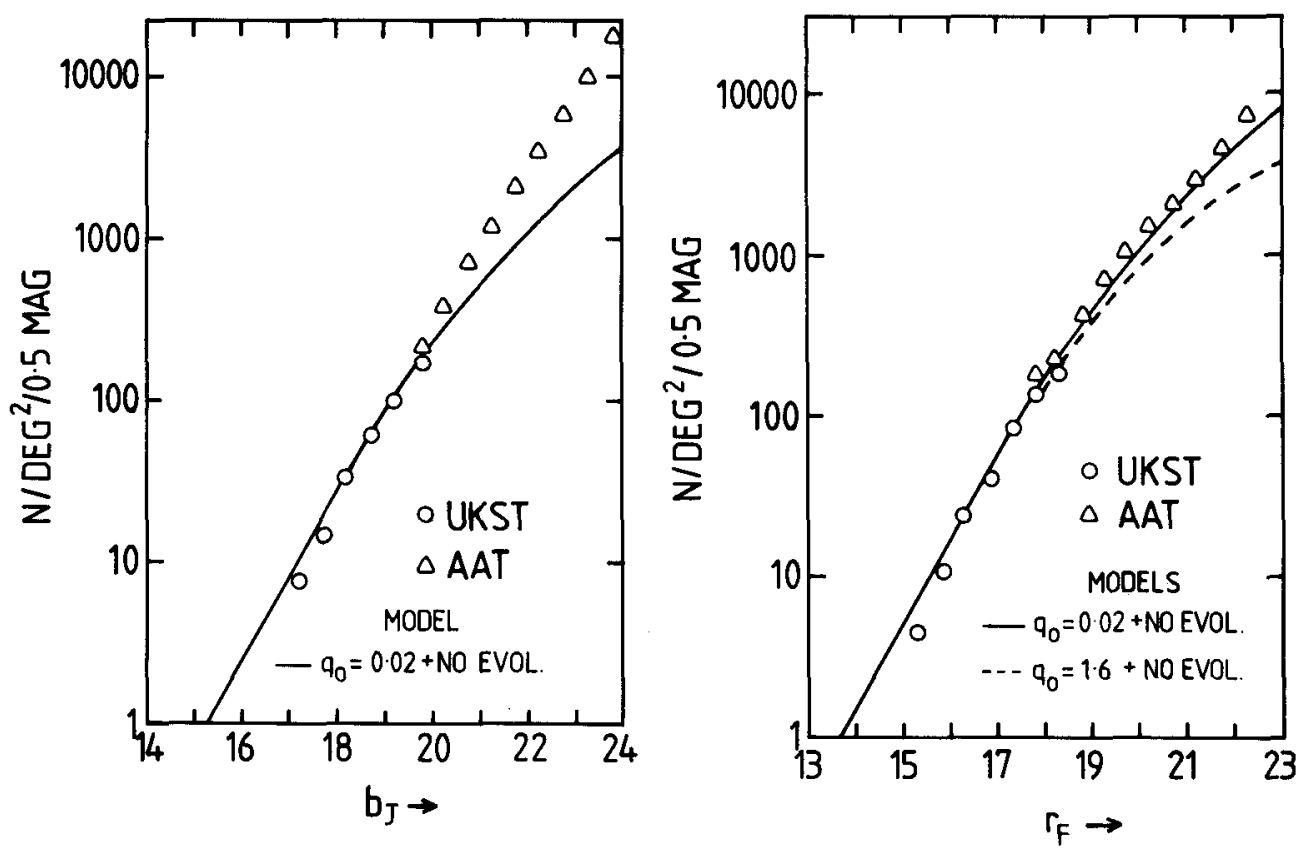

Figure 2. UKST and AAT differential galaxy number counts in the $\mathrm{b}_{\mathrm{J}}$ and $r_{F}$ bands compared to no evolution models.

these are fluctuations due to galaxy clustering because at $b_{J} \sim 23^{m}$ galaxies appear relatively unclustered on the sky due to the effect of projection. One explanation for the discrepancy could be the existence of patchy galactic obscuration in some of the fields used by Tyson and Frvis and Peterson et al. Couch and Newell measured the absorption in each of their widely separated fields from the reddening of field galaxy colours and they found that patchy absorption at lower galactic latitudes accounted for much of the dispersion in their galaxy counts.

In the red band only Kron, Koo and Couch and Newell have produced galaxy counts. When account is taken of the different red passband used by Kron and Koo we find that our faint AAT counts are in good agreement with all of these.

At the brighter magnitudes of the UKST counts, fluctuations caused by galaxy clustering may be a bigger problem. To test how typical the original 12 square degree area of SSFM was, we recently obtained COSMOS measurements on 6 adjoining UKST fields which extends our measured area at the SGP to some 110 square degrees. The resulting $b_{J}$ galaxy counts in each of these fields is shown together with the original in Figure 3. The excellent agreement among all the fields suggests that the original 12 square degree area was very representative 


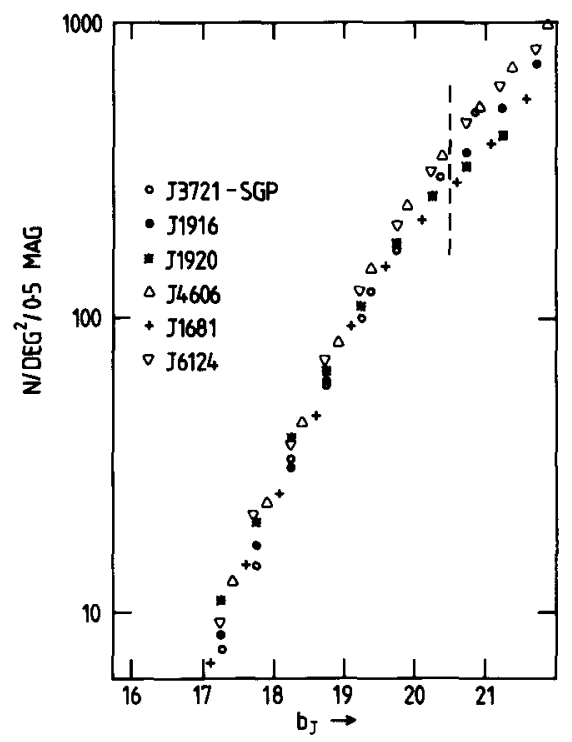

Figure 3

Galaxy counts in 6 separate UKST fields around the SGP. J3721 is the UKST plate number of the original 12 square degree field of SSFM. The dashed line indicates the $b$ $=20.5$ limit of reliability for the UKST counts.

in terms of its number count properties.

There are few independent galaxy counts in the range $17^{\mathrm{m}} \leqslant \mathrm{b}_{\mathrm{J}} \leqslant 20.5$ with which our UKST counts can be compared. However SSFM found that our counts seemed to extend naturally from those made at brighter magnitudes by Kirshner et al. (1979) and Zwicky et al. (1961-68).

\section{GALAXY COUNT MODELS}

Galaxy number count models depend chiefly on the K-corrections, the assumed mix of morphological types and the nearby galaxy luminosity function. Details of the model parameters assumed here are given by SSFM. The red models are better determined than the blue because the blue K-corrections are less reliable at high redshift and because the blue counts contain a much wider spread of morphological types than the red which are dominated by early type E/SO/Sab galaxies. We normalised our models to the UKST counts at bright magnitudes and checked that our assumed morphological mix gave good fits to the observed distribution of galaxy colours.

The solid lines in Figures $2 a$ and $2 b$ are the $q_{0}=0.02$, no evolution, standard models of SSFM. It can be seen that in the blue this model underestimates the observed count at $b_{J}=23^{m}$ by more than a factor of 3. The conclusion of SSFM was that the counts therefore implied quite large amounts of galaxy luminosity evolution in the sense that galaxies were brighter in the past.

The no evolution models of Koo (1981) adopted a fainter luminosity function for late type galaxies which decreased the average redshift of galaxies in the model and so produced a steeper number count slope. However this model predicts only a factor of 50 per cent more galaxies than ours at 23rd magnitude in $b_{J}$ and therefore still 
requires quite large amounts of evolution to fit the counts. The difference between these two models is a reasonable representation of the uncertainty in the blue model parameters and we conclude that these uncertainties may not be large enough to allow non-evolving models to explain the counts.

In the red band the $\mathrm{q}_{\mathrm{O}}=0.02$ no evolution model of SSFM gives a much better fit to the observations. This difference between the red and blue counts is expected since evolutionary models like those of Bruzual (1981) predict that galaxy luminosity will generally evolve faster at bluer wavelengths. Here the Koo (1981) model is indistinguishable from our own, indicating the smaller uncertainty associated with the red models. The dashed line in Figure $2 b$ is the $\mathrm{q}_{0}=1.6$ no evolution model which seems to give a worse fit to the observed count. This shows that the effects of $q_{0}$ on the red counts at these depths can be quite significant. Of course, world models with $\mathrm{q}_{0}=1.6$ still cannot be rejected on the basis of this result because, with the inclusion of luminosity evolution, such world models can be made to give satisfactory fits to our data.

The $\mathrm{q}_{0}$ dependence of the red counts is interesting because it is different from other cosmological tests which depend on the luminosity redshift relation. In the Hubble diagram, for instance, larger values of $q_{0}$ mimic evolutionary brightening at high redshift whereas in the number counts it is smaller values of $q_{o}$ that produce this effect. These almost orthogonal dependencies suggest that it may be possible to use the counts and the Hubble diagram together to obtain quite strong empirical constraints on both $q_{0}$ and galaxy luminosity evolution. The problem with this approach is that it assumes the evolution of cluster and field early type galaxies is the same and this assumption may be difficult to justify.

An alternative approach to the luminosity redshift relation which overcomes this problem is to determine the average redshift, $\bar{z}$, of galaxies in faint, ( $R \sim 21^{\mathrm{m}}$ ) magnitude limited samples. The advantage of this approach over the Hubble diagram is that it is selfconsistent; the same galaxies that define the $n(m)$ relation define $\bar{z}$. The disadvantage with $\bar{z}$ is that much telescope time has to be spent obtaining redshifts for many low redshift, low luminosity galaxies. However the advent of multi-object spectroscopic techniques should make this less of a difficulty and indeed a start has been made on such a project by Koo and Kron at Kitt Peak.

To test the $q_{0}$ dependence of $\bar{z}$ we have used three models that assume different combinations of $q_{0}$ and luminosity evolution but all of which produce excellent fits to the red galaxy counts. Table 1 gives the computed $\bar{z}$ for each of these models in a magnitude limited sample with $20.5 \leqslant \mathrm{r}_{\mathrm{F}} \leqslant 21^{\mathrm{m}}$. It can be seen that a relatively small redshift survey should be able to discriminate between the $q_{0}=0.02$ and $q_{0}=1.6$ models and indeed that there is quite a significant difference between the $q_{0}=0.02$ and $q_{0}=0.5$ models. 
Thus the future prospects for obtaining empirical constraints on both $\mathrm{q}_{\mathrm{O}}$ and luminosity evolution using this approach seem encouraging.

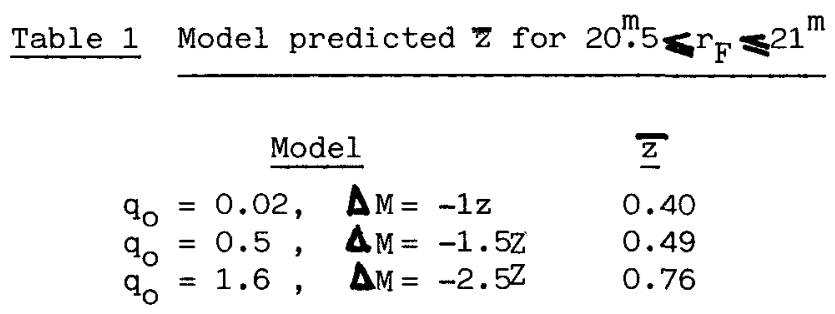

All 3 models give same predicted galaxy count as data.

$\Delta M$ is the change in absolute magnitude of galaxies at redshift $z$.

\section{CONCLUSIONS}

1. Our blue galaxy counts at the South Galactic Pole show evidence for luminosity evolution in the sense that galaxies seem to have been brighter in the past.

2. Our red galaxy counts show evidence for smaller amounts of evolution than in the blue.

3. The combination of the red counts and faint galaxy redshift surveys may produce empirical constraints on $\mathrm{q}_{0}$ and galaxy luminosity evolution.

\section{REFERENCES}

Bruzual, A.G., 1981, Ph.D. Thesis, University of California, Berkeley . Carter, D., 1980, Mon. Not. R. astr. Soc., 190, 307. Couch, W.J., and Newell, E.B., 1984 in preparation. Couch, W.J., Pence, W.D., and Shanks, T., 1984 in preparation. Fong, R., Godwin, J.G., Green, M.R., and Shanks, T. 1983 "Proc. of the Workshop on Astronomical Measuring Machines" ed. Stobie, R.S., and McInnes, B., Royal Observatory Edinburgh. Kirshner, N.P., Oemler, A., and Schechter, P.L., 1979, Astr. J. 84, 951. Koo, D.C., 1981, Ph.D. Thesis, University of California, Berkeley. Kron, R.G., 1978, Ph.D. Thesis, University of California, Berkeley. MacGillivray, H.T., and Dodd, R.J., 1982, The Observatory, 102, 141. Peterson, B.A., Ellis, R.S., Kibblewhite, E.J., Bridgeland, M., Hooley, T., and Horne, D., 1979, Astrophys. J. 233, L109. Shanks, T., Stevenson, P.R.F., Fong, R., MacGillivray, H.T., 1984, Mon. Not. R. astr. Soc. 206.

Stobie, R.S., Smith, G.M., Lutz, R.K., and Martin, R., 1979, in "Proc. of the International Workshop on Image Processing in Astronomy, Trieste, Italy.

Tyson, J.A., and Jarvis, J.F., 1979, Astrophys. J. 230, L153.

Zwicky, F., Herzog, E., Wild, P., Karpowicz, M., and Kowal C.T., 1961-1968 "Catalogue of galaxies and clusters of galaxies" in 6 vols. Calif. Inst. of Tech., Pasadena. 


\section{DISCUSSION}

C. CARTER: Have you made any counts on IV-N plates?

T. SHANKS: No, but we do have IVN UKST, AAT photographs and also I CCD frames for calibration and we intend to next make I counts using this material. The advantage of $I$ counts over $R$ counts is that we can probe to deeper redshifts before the quickly evolving ultraviolet part of ga laxy spectra is redshifted into the $I$ band. 